\title{
MODELO DE COMERCIALIZACIÓN EN CENTROS DE INVESTIGACIÓN EN EL ESTADO DE GUANAJUATO, MÉXICO
}

Carmen Castrejón Mata, Universidad de Guanajuato, coordinación Licenciatura en Mercadotecnia División de Ciencias Sociales y Administrativas.

Correo Electrónico: carsais@hotmail.com

Roberto Hernández Sampieri, Instituto Politécnico Nacional de México.

Correo Electrónico: iscelp@prodigy.net.mx

Rafael Espinosa Mosqueda: Coordinador del Doctorado Itinerante con la Universidad Nacional de Trujillo (Perú); Coordinador de la Carrera en Administración Financiera y Coordinador de la Maestría en Fiscal en el

Campus Celaya Salvatierra, Universidad de Guanajuato.

Correo Electrónico: asesorneg@yahoo.com.mx

\section{RESUMEN}

Durante varios años de llevar a cabo la investigación acerca de los factores que han incidido en la falta de comercialización de los productos desarrollados en centros de investigación en el estado de Guanajuato y basándose en la información obtenida se desarrolló una propuesta de un modelo de comercialización que lograra integrar los elementos que emergieron como categorías en las entrevistas aplicadas a responsables e investigadores y que permitiera visualizar la posibilidad de contrarrestar esos factores para lograr que esos productos pudieran satisfacer necesidades existentes en el mercado.

Palabras clave: factibilidad comercial, investigación, propiedad intelectual, productos.

JEL: M31,O14,O32.O34 


\title{
MARKETING'S MODEL OF GUANAJUATO MEXICO'S STATE RESEARCH'S CENTERS.
}

Carmen Castrejón Mata, Universidad de Guanajuato, coordinación Licenciatura en Mercadotecnia División de Ciencias Sociales y Administrativas. Correo Electrónico: carsais@hotmail.com

Roberto Hernández Sampieri, Instituto Politécnico Nacional de México. Correo Electrónico: iscelp@prodigy.net.mx

Rafael Espinosa Mosqueda: Coordinador del Doctorado Itinerante con la Universidad Nacional de Trujillo (Perú); Coordinador de la Carrera en Administración Financiera y Coordinador de la Maestría en Fiscal en el Campus Celaya Salvatierra, Universidad de Guanajuato.

Correo Electrónico: asesorneg@yahoo.com.mx

\begin{abstract}
For several years conducting research on the factors that have influenced the lack of commercialization of products developed at research centers in the state of Guanajuato and based on information obtained developed a proposed model of marketing managed to integrate the elements that emerged as categories in the interviews applied to makers and researchers, and that would display the ability to counter these factors to ensure that these products could meet requirements in the market.
\end{abstract}

Key words: comercial feasibility, research, intelectual property, products.

JEL: M31,O14,O32.034 


\section{INTRODUCCION}

De acuerdo a la Secretaría de Economía en el 2011 había más de 4 millones de empresas en México, de las cuales 99.8\% eran pequeñas y medianas empresas (PyME's), que generaban 7 de cada 10 empleos en el país y aportaban aproximadamente 50\% del PIB, según datos del Bancomext (Moreno, 2010).

En las PyME's, la innovación cobra importancia como estrategia de alto riesgo, ya que éstas no tienen la capacidad económica para reponerse si fracasan al implementar una innovación tecnológica para aumentar su productividad; sin embargo, la pasividad también las puede llevar al fracaso debido a la competencia interna y externa, por lo que resulta importante apoyarlas con productos que sean innovadores en el mercado, lo que permitiría lograr un incremento en su productividad al estar comercializando lo que realmente demanda el mercado.

\section{Tabla 1}

Productividad relativa de América Latina (países seleccionados) con respecto a la de los Estados Unidos ${ }^{1}$.

\begin{tabular}{|l|c|c|c|c|}
\hline \multicolumn{1}{|c|}{ Sector } & $\begin{array}{c}1990 \\
(\%)\end{array}$ & $\begin{array}{c}1998 \\
(\%)\end{array}$ & $\begin{array}{c}2003 \\
(\%)\end{array}$ & $\begin{array}{c}2008 \\
(\%)\end{array}$ \\
\hline Agricultura, caza, silvicultura y pesca & 14.2 & 13.3 & 10.7 & 7.0 \\
\hline Explotación de minas y canteras & 40.9 & 59.2 & 51.5 & 70.2 \\
\hline Industria manufacturera & 25.0 & 20.7 & 16.1 & 14.6 \\
\hline Electricidad, gas y agua & 23.4 & 34.4 & 31.0 & 34.8 \\
\hline Construcción & 21.0 & 22.2 & 23.7 & 33.5 \\
\hline Comercio, restaurantes y hoteles & 27.4 & 17.1 & 13.2 & 14.4 \\
\hline Transporte & 19.2 & 18.2 & 13.8 & 11.2 \\
\hline Establecimientos financieros & & 17.9 & 19.7 & 22.0 \\
\hline $\begin{array}{l}\text { Servicios comunales, sociales y } \\
\text { personales }\end{array}$ & 18.1 & 18.1 & 18.2 & 18.8 \\
\hline Total & 18.4 & 17.0 & 15.4 & 16.2 \\
\hline
\end{tabular}

1 Elaborada con datos del Estudio Económico para América Latina y el Caribe de la Comisión Económica para América Latina (CEPAL). 
De ahí la imperiosa necesidad de orientarlas y apoyarlas en la implementación de innovaciones tecnológicas: por qué innovar, qué innovar, cómo innovar, cuándo innovar, cuánto cuesta innovar, considerando las políticas de apoyo a PyME's y las políticas de innovación, para evitar reducirlas a ser maquiladoras de productos para otras empresas -sobre todo extranjeras de países desarrollados- que se quedan con grandes márgenes de ganancia sin contribuir a la riqueza de los países en desarrollo, agotando los insumos naturales y cuando no se les supervisa en forma correcta contaminando al país anfitrión (Paredes y Loyola, 2006).

Es necesario implementar más y mejores centros de creación de innovación y tecnología, encargados de desarrollar y evaluar nuevos conocimientos, soluciones, técnicas, tecnología, productos, etc., para que las PyME's aumenten su productividad y puedan sobrevivir sin problemas. Pero también es importante revisar el destino de su producción, a fin de eficientar el gasto público invertido en Ciencia y Tecnología.

\section{REVISION DE LA LITERATURA}

En México los centros de innovación y tecnología son conocidos como centros de investigación, cuyo objetivo es entregar a las PyME's un producto terminado y probado, ya sea un conocimiento, una técnica, una tecnología, la producción de un bien, etc., para que la PyME lo implemente sin contratiempos y se refleje en la productividad del país. Estas instituciones de investigación también deben considerar las necesidades de las grandes empresas del sector privado y público, así como de organismos exportadores.

Desafortunadamente la eficacia de estos centros se ha visto afectada por diversos factores (laborales, políticos, económicos, etc.), que repercuten en su capital intelectual.

Comparado con países desarrollados, en México hay muy pocos recursos humanos para la creación de centros de tecnología como se muestra en la figura 1. Por ejemplo, Estados Unidos -vecino y principal socio comercial- nos supera en más de 14 veces con su densidad de investigadores, esto explica los grandes pagos por tecnología hacia el exterior y muestra la necesidad de recursos humanos que identifiquen las necesidades de productos y servicios en el mercado, para satisfacer la demanda.

De acuerdo a Paredes y Loyola (2006), los problemas de infraestructura y recursos humanos para la investigación, están asociados a cuatro grandes factores que afectan en gran medida la creación, desarrollo y eficacia de los centros de tecnología: 


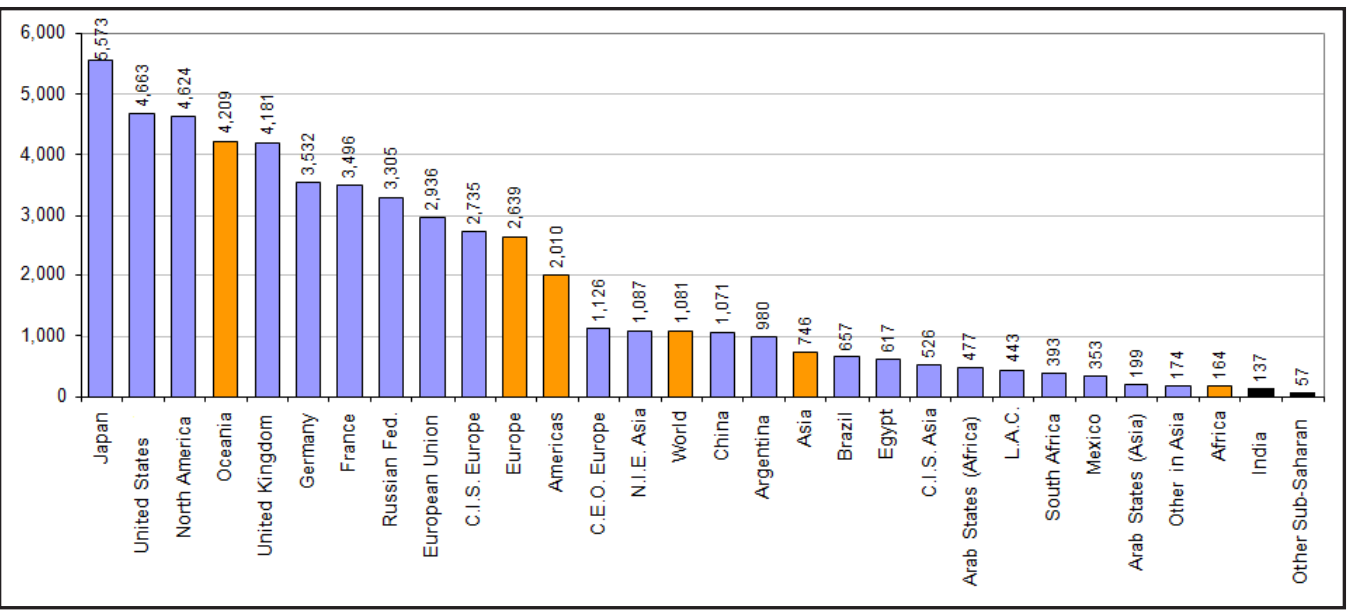

Figura 1

Investigadores por millón de habitantes de las principales regiones/países, 2007 o último año disponible ${ }^{2}$.

- Limitado gasto público en investigación y desarrollo con respecto al Producto Interno Bruto (PIB).

En México, el gasto público en investigación y desarrollo con respecto al PIB nunca ha sido mayor de 0.4\%, en tanto que, en el año 2002, en Alemania fue de 2.51, en Estados Unidos de 2.67, en Francia de 2.2, en Inglaterra 1.89, en Suecia 4.27, en Japón 3.06 y en España de 0.96 (Paredes y Loyola, 2006).

Dicho de otra manera mientras los países líderes incrementan su gasto o lo mantienen en porcentajes históricos, México lo disminuye.

- Coeficiente de inventiva y registro de patentes.

En materia de patentes y de innovación según Paredes y Loyola (2006) los resultados también dejan que desear. Existe un creciente saldo negativo en la balanza de pagos por tecnología y en el coeficiente de inventiva de México, sin hablar del peso de las patentes que se registran en México en relación con las otras economías.

El tema de la propiedad intelectual tiene que estar plenamente incorporado a la estrategia de acumulación de capacidades para el desarrollo. La región se encuentra muy atrasada en lo que

2 Adaptado del documento sobre Human resources in R\&D del Institute for Statistics, United Nations Educational Scientific and Cultural Organization (UNESCO) (2010). 
concierne al análisis de estos problemas. Sin duda, invertir en recursos humanos y en infraestructura para administrar de forma eficaz la propiedad intelectual tiene un costo elevado, que está fuera del alcance de muchos países en desarrollo y, en particular, de las pequeñas empresas.

- Acceso al financiamiento y mercado

Inexistencia de una política y falta de interés en el modelo de centros públicos de investigación. En la iniciativa de ley que derivó en la de ciencia y tecnología, se pretendió dispersar a los centros públicos de investigación; por otra parte, si bien entre el 2000 y el 2004 el presupuesto asignado pasó de 2,767 a 2,883 millones de pesos pero en términos reales hubo un decremento por el incremento de la inflación y el aumento al presupuesto del Consejo Nacional de Ciencia y Tecnología (CONACYT), además de que se perdieron los fondos acordados por la Cámara de Diputados (Paredes y Loyola, 2006).

\section{- Disponibilidad de infraestructura y tecnología.}

El progreso técnico ocurre con diferente velocidad en los distintos sectores de cada país y la tecnología y las oportunidades de innovación no se distribuyen de manera uniforme. En América Latina, estas diferencias son mucho mayores que en las economías desarrolladas y los sectores y agentes rezagados no tienden a aproximarse a los líderes (Paredes y Loyola , 2006).

Aun cuando la mayoría de las actividades tienen el propósito de dirigir el flujo de bienes y servicios del productor al consumidor, el proceso de mercadotecnia empieza por un análisis del consumidor, incluso antes de que el producto se elabore. Por ello es importante que en los laboratorios tecnológicos y de investigación se planifique el desarrollo del producto y las modificaciones al mismo para responder a los cambios en la demanda.

El CONACYT (2010) señala que se ha buscado descentralizar las actividades científicas, tecnológicas y de innovación, desarrollando así un marco estructural de los sistemas estatales de ciencia y tecnología, siendo este el siguiente:

a) Consejo Estatal de Ciencia y Tecnología.

El 23 de febrero de 1996, mediante Decreto Gubernativo número 19, se creó el Consejo de Ciencia y Tecnología del Estado de Guanajuato (CONCYTEG), como organismo público descentralizado de la Administración Estatal con personalidad jurídica y patrimonio propios. Posteriormente, con la entrada en vigor de la Ley de Fomento a la Investigación Científica, Tecnológica y a la Innovación para el Estado de Guanajuato, se ratifica la existencia del Consejo, que conserva su naturaleza jurídica, y no está sectorizado. 


\section{b) Ley de Ciencia y Tecnología.}

La Ley de Fomento a la Investigación Científica, Tecnológica y a la Innovación para el Estado de Guanajuato fue emitida mediante Decreto número 115, en el Periódico Oficial de fecha 17 de mayo de 2002, la cual entró en vigor al día siguiente de su publicación; asimismo mediante Decreto gubernativo número 125 publicado el 20 de agosto de 2002 se emitió el Reglamento de dicha Ley.

c) Planes y Programas de Ciencia y Tecnología.

El Estado de Guanajuato cuenta con un Plan de Ciencia y Tecnología 1998-2020, un Programa de Ciencia y Tecnología 2030, independientemente de que en su Plan Estatal de Desarrollo 2006 -2012, se establecen las líneas de acción en materia de ciencia, tecnología e innovación.

d) Comisión Legislativa de Ciencia y Tecnología.

El Congreso del Estado cuenta con una Comisión de Educación, Ciencia y Tecnología Cultura. e) Sistema de Información Nodal de CONACYT.

Se ha comenzado a trabajar en la conformación del Sistema de Información Nodal SIICYT-CONCYTEG, se firmó convenio de colaboración el 8 de octubre del 2010, actualmente se cuenta a nivel nacional con 20 convenios Estatales; nueve portales son consultables en internet,.

El Fondo Mixto CONACYT-Gobierno del Estado de Guanajuato fue constituido en octubre del 2001 y según el CONACYT (2010) al término de 2010, ha publicado 24 convocatorias, en las que se han aprobado 557 proyectos que representan apoyos del orden de 472 millones de pesos. De los proyectos aprobados se encuentran vigentes 160 , orientados principalmente a la modalidad de investigación científica.

Además como menciona Ramírez (2009) que es la tecnología la que nos permite saber cómo aprovechar las cosas, mejorarlas o transformarlas para beneficio de la humanidad. Con la palabra "cosas" se refiere a la naturaleza, materiales, energía y en general, a cualquier ente que puede ser estudiado y utilizado para el beneficio de la humanidad.

La empresa es el actor ejecutor que utiliza a la ciencia y a la tecnología para aprovechar y mejorar las cosas en beneficio de la sociedad y medio ambiente, el emprendedor es la persona que se anima a diseñar y llevar a cabo nuevas ideas y proyectos donde el sentido común y el pensamiento de sistemas lleva a mantener equilibrio entre ideas y proyectos, economía, sociedad y medio ambiente.

Según la OCDE (2010), la investigación y el desarrollo experimental comprenden el trabajo creativo llevado a cabo de forma sistemática para incrementar el volumen de conocimientos, 
incluido el conocimiento del hombre, la cultura y la sociedad, además del uso de esos conocimientos para derivar nuevas aplicaciones. El término I+D engloba tres actividades: investigación básica, investigación aplicada y desarrollo experimental.

- Investigación básica: consiste en trabajos experimentales o teóricos que se emprenden fundamentalmente para obtener nuevos conocimientos acerca de los fundamentos de fenómenos y hechos observables, sin pensar en darles ninguna aplicación o utilización determinada.

- Investigación aplicada: consiste también en trabajos originales realizados para adquirir nuevos conocimientos; sin embargo, está dirigida fundamentalmente hacia un objetivo práctico específico.

- Desarrollo experimental: consiste en trabajos sistemáticos basados en los conocimientos existentes, derivados de la investigación y/o la experiencia práctica, dirigidos a la producción de nuevos materiales, productos o dispositivos; al establecimiento de nuevos procesos, sistemas y servicios, o a la mejora sustancial de los ya existentes.

En los procesos de comercialización resulta importante considerar que para contrarrestar

E\&A

eficientemente el alza en los costos operativos que puedan tener las empresas, costos en el desarrollo tecnológico y otros, como lo menciona Moreno (2010) lo ideal es encaminarse en estrategias que aumenten la productividad mediante el uso de las nuevas tecnologías, modelos de gestión como el outsourcing y prácticas estratégicas de desarrollo de capital, como aconsejan los especialistas.

Fernández y Avella (2003) nos dicen que la comercialización de productos o servicios involucra gran cantidad de actividades del área de mercadotecnia, que deben tener una dirección clara, de tal forma que sus esfuerzos tengan resultados reales para la empresa.

\section{METODOLOGIA}

La primera parte de la investigación era identificar los factores que han limitado la comercialización de los productos desarrollados en los centros de investigación tecnológica en el estado de Guanajuato pues algunos de estos se encuentran aún en procesos de desarrollo en el laboratorio pero no se llevan a la industrialización o producción a escala para su comercialización, posteriormente fue identificar los elementos necesarios para desarrollar una propuesta de modelo para la comercialización de los productos desarrollados en centros de investigación tecnológica en el estado de Guanajuato que facilite y dinamice este proceso de comercialización. 
Para la selección de la muestra se consideraron investigadores que trabajan en centros de investigación en el estado de Guanajuato.

El estudio tuvo un enfoque cualitativo, los participantes en la muestra fueron investigadores que trabajan en los centros de investigación en el estado de Guanajuato, (si es posible, señalar con qué criterios se seleccionaron los investigadores), cuya constante participación en proyectos dificultó las entrevistas a profundidad, de ahí que solamente se entrevistó a ocho investigadores de los centros de investigación. Adicionalmente se obtuvieron datos cuantitativos a partir de la aplicación de un instrumento a 48 investigadores con proyectos en centros de investigación del estado citado.

\section{RESULTADOS DE LA INVESTIGACION}

Algunos de los resultados que se obtuvieron y que permitieron el desarrollo del modelo de comercialización fueron los siguientes:

El sector público solamente de una a tres veces ha tenido en un $62.5 \%$ interés por el proyecto, por esto es importante implementar no solamente políticas económicas sino programas que fomenten la comercialización. Establecer convenios entre las empresas y los centros de investigación, que éstos puedan desarrollar la autosuficiencia al promover sus productos y aquéllas sean más competitivas.

El $43.8 \%$ de los investigadores, nunca ha evaluado la factibilidad comercial de su proyecto, lo que representa un considerable porcentaje, ya que es casi la mitad de ellos, además el $45.8 \%$ lo ha hecho solamente de una a tres veces y únicamente el 10.4\% ha hecho la evaluación de factibilidad comercial, de ahí deducimos que no existe interés por la comercialización para poder satisfacer las necesidades existentes en el mercado, además es importante para definir y establecer la asignación del precio en el mercado.

En el $64.6 \%$ de los casos se carece de personal calificado para el desarrollo de su proyecto, este alto porcentaje puede impactar en la calidad del producto, el desarrollo, la permanencia en el centro de investigación y el aumento del stock impacta los costos de inventario, etc., lo que puede afectar la participación en el mercado por la discrepancia espacial y la entrada de la competencia.

El hecho de que el 39.6\% de los investigadores haya tenido problemas con el apoyo financiero es determinante para conquistar la plaza (constructo definido) en la cual se va a ofrecer el producto, facilitar la participación en el mercado al usar herramientas de la mercadotecnia, que permiten que el consumidor tenga el producto en el lugar y el momento adecuado. 
El $16.7 \%$ de los investigadores nunca ha revisado en el mercado la existencia de aplicaciones prácticas o comerciales de proyectos similares al suyo. Además, solamente el 31.3\% lo ha hecho en cuatro o más veces, esto significa una desventaja, por el escaso conocimiento de los productos de la competencia, y por las repercusiones de esto en la asignación de precios de los bienes y servicios. Algunos de los aspectos que deben considerar los centros de investigación son: que los proyectos desarrollados correspondan a una necesidad, utilización y aplicación bien definida con apoyo del área de evaluación y actualización tecnológica, considerando al área de diseño industrial para facilitar la comercialización; que puedan satisfacer las expectativas de los consumidores tanto individuales como industriales según el caso; que sean acordes a normas y especificaciones aplicables, por eso se canalizarán al área de asesoría legal, además es importante que estén de acuerdo a requisitos reglamentarios y ser accesibles para la sociedad por sus precios competitivos a través de la evaluación y factibilidad comercial.

Lo anterior nos llevó a construir la siguiente propuesta:

E\&A

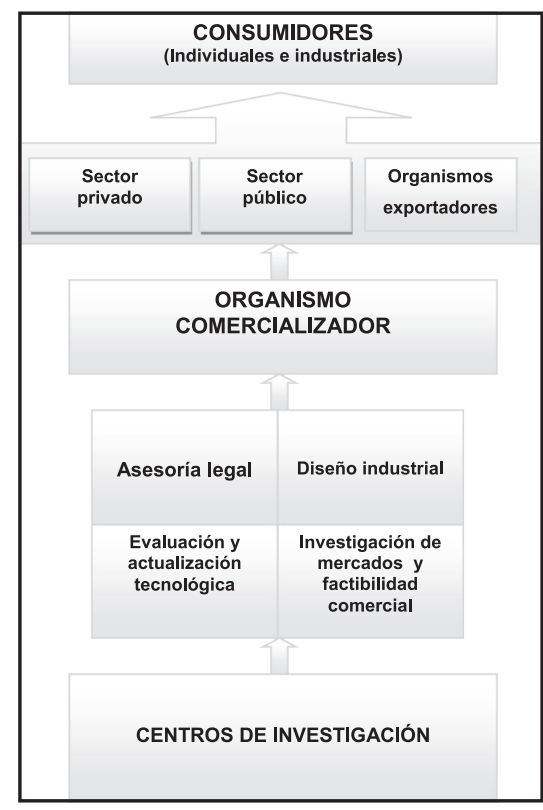

Figura2

Modelo de comercialización para los productos desarrollados en centros de investigación ${ }^{3}$.

3 Modelo propio desarrollado a partir de la información obtenida durante la investigación . 
La evaluación y actualización tecnológica, podrá apoyar a que el producto tenga las características y elementos necesarios para hacerlo competitivo en el mercado, pues el éxito o fracaso en éste, a veces dependen de especificaciones muy particulares y el retraso en el desarrollo de los proyectos puede ser un factor determinante para entrar antes que la competencia haga su lanzamiento.

Dosal, Gutiérrez y Saracho (2011) mencionan que hay distintas tendencias en la asignación de apoyos gubernamentales para fomentar la innovación, según el nivel de desarrollo de un país. Por un lado, en países como Canadá, seis de cada diez plantas manufactureras reciben algún apoyo, siendo los más comunes los incentivos fiscales. Así mismo, más de un tercio de las empresas innovadoras en Noruega, Italia, Holanda y Finlandia reciben apoyos públicos para fomentar la innovación. En el caso mexicano, el $60.7 \%$ de las empresas mencionan la falta de apoyos públicos como un obstáculo a la innovación. No obstante, cerca de un $20 \%$ de las empresas innovadoras considera estos apoyos como la segunda fuente de financiamiento.

En la evaluación y factibilidad comercial se deberá analizar la demanda de los productos desarrollados, los similares o sustitutos que existen en el mercado o en su caso buscar la información necesaria de aquellos que vaya introducir la competencia, además de conocer no solamente el mercado local o regional, sino el nacional e internacional si es necesario. Así también la estimación de estrategias que permitan pasar al proceso de comercialización y establecimiento de precios a través de la estimación de los insumos requeridos y del establecimiento de las economías de escala de los productos.

Sin embargo, Espinoza (2004) dice que según Salter la dificultad en las innovaciones y la tecnología está en la "traducción" del conocimiento que debe ser comercialmente posible, es decir, que la esperanza de sus retornos sea económicamente positiva, para lo cual deben trabajar en las distintas alternativas de costos inherentes a la nueva técnica de producción. El conjunto de técnicas disponibles para las empresas está limitado por la existencia de las máquinas necesarias para tales fines y el costo de los insumos requeridos por los productos de la innovación. 
La asesoría legal, tiene que estar plenamente incorporada a la estrategia con el tema de la propiedad intelectual porque además garantiza la competencia en el mercado a través de la obtención de patentes y marcas, manejo de contratos, elaboración de proyectos, etc.

Para apoyar la estrategia del desarrollo de los proyectos en productos para la comercialización se apoyará con el área de diseño industrial porque de acuerdo a los resultados de la investigación, la falta de motivación para el desarrollo del los proyectos puede impactar en el desarrollo de la creatividad para la generación de ideas que se transformen en productos para el mercado, además el diseño que busca crear o modificar objetos o ideas para hacerlos útiles, prácticos o simplemente bellos no solo en su forma sino también en las funciones.

Así mismo, más de un tercio de las empresas innovadoras en Noruega, Italia, Holanda y Finlandia reciben apoyos públicos para fomentar la innovación. En el caso mexicano, el 60.7\% de las empresas mencionan la falta de apoyos públicos como un obstáculo a la innovación. Por esto, resulta importante dentro del modelo teórico propuesto gestionar los apoyos necesarios para la actualización tecnológica, ya que de acuerdo a Espinoza (2004) este nuevo conocimiento es difundido en la empresa y deriva en la producción de un nuevo bien o en la mejora en los procesos productivos de un bien ya existente.

El organismo comercializador tendrá el contacto directo con el sector privado, sector público y organismos exportadores. Llevará a cabo las actividades de mercadotecnia necesarias para el proceso de comercialización de los productos, identificando las áreas de oportunidad existentes a través de la investigación de mercados, para que los productos que sean desarrollados en los centros de investigación lleguen como respuesta a las cambiantes necesidades y deseos de los consumidores. En este proceso de comercialización derivado intervienen muchos elementos que van a fortalecer esa relación productor-consumidor. Además, ya que una de las tareas más importantes de la mercadotecnia es la de identificar e interpretar constantemente esos cambios que se dan en los consumidores antes de llevar a cabo el proceso de producción. Aun cuando la mayoría de las actividades tienen el propósito de dirigir el flujo de bienes y servicios del productor al consumidor, el proceso de mercadotecnia empieza por un análisis del consumidor e incluso antes de que el producto se elabore, por eso la relación directa de este organismo comercializador con los sectores que los canalizan para entregar bienes y servicios a los consumidores quieren y necesitan, haciéndoselos llegar en el momento oportuno, en el lugar adecuado y al precio que estén dispuestos a pagar. 
El sector privado este sector busca estimular el crecimiento económico a través de las inversiones para crear empleos productivos y aumentar los ingresos. En este sector podremos encontrar a empresas de todo tipo como manufactureras, de servicios, etc., que requieren de maquinaria o producto terminado. En el modelo propuesto será un importante enlace entre el organismo comercializador y los consumidores porque contribuye con la aportación de capital en la comercialización y en la generación de utilidades para la re-inversión.

Dosal et al. (2011) explican que ante la falta de acceso a financiamientos en el sector privado, los programas y fondos gubernamentales, tanto federales como estatales, representan la segunda fuente de financiamiento para las empresas innovadoras, sólo superados por los recursos propios. Más de un tercio de las empresas que ellos estudiaron expresaron que el apoyo gubernamental fue clave durante alguna de sus etapas de desarrollo. Aún cuando las empresas mencionaron la importancia del papel que ha tenido el apoyo gubernamental para innovar.

El sector público como todos sabemos adquiere los bienes o servicios para llevar a cabo sus principales funciones, principalmente de tipo social: como drenaje, pavimentación, limpieza, compra muebles, equipo de oficina, combustible, papelería, etc., compra una gran cantidad de productos que considera necesarios para lograr el mantenimiento de la sociedad, así como de las instituciones públicas como las universidades u otros organismos públicos que requieren una serie de insumos que posteriormente ofrecen a los ciudadanos transformados principalmente en servicios.

Dosal et al. (2011) mencionan que de acuerdo a la OCDE los apoyos gubernamentales destinados al fomento de la innovación tienen un impacto considerable en empresas que se encuentran tanto cerca como lejos de la frontera tecnológica. Aquéllas que están lejos de la frontera y reciben apoyos gubernamentales, gastan entre 60\% y 100\% más en innovación que las empresas que no reciben fondos. Para las empresas cercanas a la frontera tecnológica, se destina un 30-50\% más en actividades de este tipo, además considerando esto como lo menciona Espinoza (2004), la idea es proponer a través del análisis de los determinantes de la inversión en $\mathrm{I}+\mathrm{D}$, políticas de incentivos a nivel microeconómico que permitan a las empresas ser más competitivas, de forma tal, que participen de los beneficios que genera la globalización. 
Los organismos exportadores, son indispensables para el proceso de internacionalización de un país, la promoción a través de estos sectores permite la vinculación para que lleve a cabo la promoción de los proyectos cuyas innovaciones también pueden acceder a mercados internacionales: innovar en productos y/o servicios que son versiones mejoradas de las ya existentes, pueden ofrecer ventajas comparativas a las empresas mexicana (Dosal et al., 2011).

Además, bajo el actual modelo secundario exportador, México es un país con uno de los niveles de vinculación de negociación comercial más elevado del mundo. En una economía cerrada, el consumidor no tiene posibilidades de elección en materia de precio y calidad, por lo que como productores de bienes y servicios, estamos obligados a innovar y desarrollar cada vez más y mejores productos. La exportación debe formar parte importante de la planeación estratégica y de crecimiento no sólo de las empresas, sino de los centros de investigación, pues las reglas han cambiado como resultado de la mayor disponibilidad de productos y servicios para el consumidor.

Los consumidores (individuales $\boldsymbol{e}$ industriales) no son un simple agente pasivo que espera a que le ofrezcan los productos y servicios, son agentes activos con el poder suficiente para lograr cambios en las ofertas y hasta en las mismas empresas, para que se ajusten a sus requerimientos y necesidades, que pueden ser generadores de ideas para el desarrollo en los centros de investigación.

Proponemos el desarrollo de las especificaciones y particularidades para cada uno de los proyectos dentro del Modelo de comercialización para los productos desarrollados en centros de investigación, además del conocimiento de los perfiles específicos de los consumidores de los productos y servicios, para facilitar y dinamizar la comercialización de los productos de los centros de investigación. Ésta es un área de oportunidad para la generación de recursos y el proceso de sustentabilidad de los propios centros.

La OCDE lanzó el Proyecto de Microdatos en Innovación en el cual participaron equipos de investigación de 21 países. A partir de los microdatos sobre innovación disponibles para cada país, se estimó la relación entre innovación y productividad (Dosal et al., 2011).

Al analizar el modelo de comercialización desarrollado en la presente investigación se ha llegado a reflexiones importantes independientemente de los resultados de la investigación y se exponen en los resultados del Proyecto de Microdatos que fueron (Dosal et al., 2011): 
- Las empresas con operaciones en mercados internacionales, que reciben apoyos gubernamentales y que tienen actividades de colaboración con otras instituciones invierten más recursos en actividades innovadoras que otras empresas.

- Las empresas que asignan un mayor gasto en proyectos de innovación y que introducen innovaciones tanto en productos como en proceso obtienen más utilidades.

- Las empresas con una mayor intensidad en ventas debido a una innovación tienen mayores niveles en productividad.

Además, es importante identificar si la distribución de los presupuestos para la investigación es suficiente y correctamente aplicada porque muchas veces éstos se utilizan más para la evaluación de los investigadores (gastos administrativos), que para el propio desarrollo y evaluación de la investigación, así como la comercialización de los productos de investigación.

Es necesario investigar si existen elementos determinantes entre la innovación y aspectos desde el punto de vista del origen de formación de los investigadores (instituciones públicas o privadas). Además es necesario evaluar el papel que juega en el aprovechamiento de las innovaciones el organismo regulador de la propiedad industrial (IMPI), en términos de burocratización de los procesos o afectación económica a las empresas.

Se puede identificar el papel de los investigadores en el proceso de comercialización como generadores de negocio y analizar la importancia del plan de negocios en el proceso de comercialización de los productos de los centros de investigación, de qué manera afecta el contexto tecnológico, económico, legal y socio-cultural de acuerdo al estudio de mercado, así como identificar si el proyecto es un negocio escalable y analizar cuánta inversión se requiere.

La aplicación del modelo en el proceso de comercialización puede ser una alternativa no solo para satisfacción de necesidades del mercado sino además para la obtención de recursos.

\section{CONCLUSIONES}

Con el desarrollo de la investigación se confirmaron los supuestos de que debido a su rol principal, los investigadores no saben de comercialización, no tienen tiempo para revisar patentes, no saben y/o no tienen tiempo para bajar recursos etc., de ahí la importancia de un organismo comercializador. 
A partir del análisis de los datos obtenidos en la investigación, se desarrolló un modelo de comercialización para los productos desarrollados en centros de investigación, que considera los factores que limitan dicho proceso para los productos desarrollados en los centros de investigación en el estado de Guanajuato, a fin de que éstas instituciones puedan incrementar sus ingresos y lograr una mayor satisfacción de necesidades en el mercado.

En el modelo propuesto se incluyen sus componentes: el aspecto legal, de diseño industrial, así como otros elementos para agilizar los procesos.

Algunos elementos que se deben considerar para proporcionar el apoyo a los centros de investigación de acuerdo al modelo son: que los proyectos desarrollados correspondan a una necesidad, utilización y aplicación bien definida con apoyo del área de evaluación y actualización tecnológica, considerando al área de diseño industrial para facilitar la comercialización; que puedan satisfacer las expectativas de los consumidores individuales como industriales según sea el caso; que estén de acuerdo a normas y especificaciones aplicables, por eso se canalizarán al área de asesoría legal, además es importante que estén de acuerdo a requisitos reglamentarios de la sociedad; deberán de buscar que estén disponibles a precios competitivos a través de la evaluación y factibilidad comercial.

Además, es importante identificar si la distribución de los presupuestos para la investigación es suficiente y correctamente aplicada, porque muchas veces éstos se utilizan más para la evaluación de los investigadores (gastos administrativos), que para el propio desarrollo y evaluación de la investigación o la comercialización de los productos de investigación.

Los productos requieren de especificaciones muy particulares, la evaluación y actualización tecnológica, favorecerá la inserción exitosa de la producción de los centros de investigación en el mercado antes que la competencia haga su lanzamiento.

Es importante el incremento de los recursos para fomentar la innovación, ya que en el caso mexicano, el $60.7 \%$ de las empresas mencionan la falta de apoyos públicos como un obstáculo en este rubro. No obstante, cerca de un $20 \%$ de las empresas innovadoras considera estos apoyos como la segunda fuente de financiamiento. 
En la evaluación y factibilidad comercial se deberá investigar y analizar la demanda de los productos desarrollados, los similares o sustitutos que existen en el mercado y no solamente en el mercado local o regional, sino además en el nacional e internacional si es necesario. Así también la estimación de estrategias que permitan pasar al proceso de comercialización y establecimiento de precios a través de la estimación de los insumos requeridos y del establecimiento de las economías de escala de los productos.

Dicho análisis debe considerar las limitaciones de infraestructura e insumos para la innovación de productos.

La asesoría legal, tiene que estar plenamente incorporada a la estrategia con el tema de la propiedad intelectual porque además garantiza la competencia en el mercado a través de la obtención de patentes y marcas, manejo de contratos, elaboración de proyectos, etc., se apoyará con el área de diseño industrial porque de acuerdo a los resultados de la investigación, la falta de motivación para el desarrollo del los proyectos puede impactar en el desarrollo de la creatividad para la generación de ideas que se transformen en productos para el mercado.

Es importante dentro del modelo teórico propuesto gestionar los apoyos necesarios para la actualización tecnológica, ya que de acuerdo a Espinoza (2004) este nuevo conocimiento es difundido en la empresa y deriva en la producción de un nuevo bien o en la mejora en los procesos productivos de un bien ya existente.

Considerando que el proceso de mercadotecnia empieza por un análisis del consumidor, incluso antes de que el producto se elabore, el organismo comercializador tendrá el contacto directo con el sector privado, sector público y organismos exportadores. Identificando las áreas de oportunidad existentes a través de la investigación de mercados, para que los productos que sean desarrollados en los centros de investigación lleguen como respuesta a las cambiantes necesidades y deseos de los consumidores haciéndoselos llegar en el momento oportuno, en el lugar adecuado y al precio que estén dispuestos a pagar. 
El sector privado en el modelo propuesto será un importante enlace entre el organismo comercializador y los consumidores porque contribuye con la aportación de capital en la comercialización y en la generación de utilidades para la re-inversión.

El sector público, para cumplir con sus funciones, compra una gran cantidad de productos, por lo cual su papel es importante en la estrategia de comercialización.

Los apoyos gubernamentales destinados al fomento de la innovación tienen un impacto considerable, principalmentre en las empresas que se encuentran lejos de la frontera tecnológica. Por ello se requieren políticas de incentivos a nivel microeconómico que permitan a las empresas ser más competitivas, de forma tal, que participen de los beneficios que genera la globalización. Los organismos exportadores, son indispensables para acceder a mercados internacionales, su vínculo con los organismos innovadores puede ofrecer ventajas comparativas a las empresas mexicana (Dosal et al., 2011).

Los consumidores (individuales $\boldsymbol{e}$ industriales) no son un simple agente pasivo que espera a que le ofrezcan los productos y servicios, son agentes activos con el poder suficiente para lograr cambios en las ofertas y hasta en las mismas empresas, para que se ajusten a sus requerimientos y necesidades, que pueden ser generadores de ideas para el desarrollo en los centros de investigación.

Se plantea como propuesta el desarrollo de las especificaciones y particularidades para cada uno de los proyectos dentro del Modelo de comercialización para los productos desarrollados en centros de investigación, además del conocimiento de los perfiles específicos de los consumidores de los productos y servicios, para facilitar y dinamizar la comercialización de los centros de investigación siendo un área de oportunidad para la generación de recursos y el proceso de sustentabilidad de los propios centros.

La OCDE lanzó el Proyecto d Microdatos en Innovación en el cual participaron equipos de investigación de 21 países. A partir de los microdatos sobre innovación disponibles para cada país, se estimó la relación entre innovación y productividad (Dosal et al., 2011).

Independientemente de los resultados de la investigación y como se expone en los resultados del Proyecto de Microdatos, el modelo de comercialización desarrollado en esta permite las reflexiones siguientes:

Los apoyos gubernamentales impactan positivamente la innovación, a su vez esto mejora la productividad y las utilidades. 
Sin embargo, es importante identificar si la distribución de los presupuestos para la investigación es suficiente y correctamente aplicada porque muchas veces éstos se utilizan más para la evaluación de los investigadores (gastos administrativos), que para el propio desarrollo y evaluación de la investigación, así como la comercialización de los productos de investigación.

Es necesario investigar si existen elementos determinantes entre la innovación y el origen de formación de los investigadores (instituciones públicas o privadas), y evaluar el papel que juega en el aprovechamiento de las innovaciones el organismo regulador de la propiedad industrial (IMPI), en términos de burocratización de los procesos o afectación económica a las empresas.

Se puede identificar el papel de los investigadores en el proceso de comercialización como generadores de negocio y analizar la importancia del plan de negocios en el proceso de comercialización de los productos de los centros de investigación, de qué manera afecta el contexto tecnológico, económico, legal y socio-cultural de acuerdo al estudio de mercado, así como identificar si el proyecto es un negocio escalable y analizar cuánta inversión se requiere.

La aplicación del modelo en el proceso de comercialización puede ser una alternativa no solo para satisfacción de necesidades del mercado sino además para la obtención de recursos, por parte de los centros de investigación, lo cual podría eficientar el gasto público. 


\section{LISTA DE REFERENCIAS}

Aboites, J. (2009).Generación de conocimiento tecnológico y negocios. Ide@s CONCYTEG, 50(4), 846-858.

Aguirre de Lázaro, E., Rodríguez, C. y Romero, O. (2004). Indicadores de actividades científicas y tecnológicas para la evaluación institucional de centros de educación superior en Cuba, en los inicios del siglo XXI. La Habana, Cuba.

Brache, A. y Bodley-Scott, S. (2006). Cómo transformar las iniciativas estratégicas en resultados arrasadores.Implementación. (1a. Ed. ). México, DF, México: Mc Graw Hill.

Centro de Investigación Científica y de Educación Superior de Ensenada (CICESE).Comercialización de la ciencia y la tecnología (2010). Recuperado de: http://gaceta.cicese.mx/print.php?topico=secciones\&ejemplar $=97 \&$ sid $=\& i d=2249$

Churchill, G. A. (2003). Investigación de mercados (4a. ed.). México, DF, México: Thomson.

Comisión Económica para América Latina (2010) . Estudio económico para América Latina y el Caribe. Recuperado el 22 de noviembre de 2010, de: http://www.eclac.org

Consejo de Ciencia y Tecnología del Estado de Guanajuato (2010). Página web sobre servicios, centros de investigación y observatorio de ciencia y tecnología. Recuperado el 19 de noviembre de 2010, de: http://www.concyteg.gob.mx/index.html

Consejo Nacional de Ciencia y Tecnología (2010). Página web. Recuperado el 12 de febrero de 2010, de: http://www.conacyt.mx

Dosal, C., Gutiérrez, C. y Saracho, A. (2011). ¿Quiénes son los emprendedores innovadores mexicanos?. México: Fundación IDEA, A.C. 
Espinoza, H. (2004).Inversión en investigación y desarrollo para generar competitividad. (2004). Recuperado el 18 de noviembre de 2010, de: www.cepes.org.pe/apc-aa/archivos-aa/.../pba_01.pdf

Fernández, E. y Avella, L. (2003). Estrategias de producción. Barcelona, España: Mc Graw Hill.

Global Competitiveness Report 2010-2011 (2010). Indice Global de Competitividad 2010-2011. Recuperado el 24 de abril de 2011, de: http:/www.weforum.org/issues/global-competitiveness

Gobierno del Estado de Guanajuato (2007). Impulsamos la ciencia y el desarrollo tecnológico. (s.f.). Recuperado el 9 de agosto de 2007, de: http://lideres.guanajuato.gob.mx

Hernández, R., Fernández, C. y Baptista, P. (2006). Metodología de la investigación (4a. ed.). México, DF, México: Mc Graw Hill.

Human resources in R\&D. Institute for Statistics. (2010). Recuperado el 24 de abril de 2011, de:http:// www.uis.unesco.org/ev.php?URL_ID=3755\&URL_DO=DO_TOPIC\&URL_S ECTION=201

Instituto Mexicano de la Propiedad Industrial (2010). Página web. Recuperado el 19 de noviembre de 2010, de: www.impi.gob.mx

Instituto Politécnico Nacional (2004). Manual para consultores en mercadotecnia. Guadalajara, Jalisco, México: Instituto de Estudios Superiores de Occidente.

Moreno, T. (2010). CNNEXPANSION. Recuperado de 19 de noviembre de 2011, de: http://www. cnnexpansion.com/emprendedores/2010/01/28/4-tips-que-elevan- la-competitividad-pyme.

Nieto, J. (2008).Y tú...,¿innovas o abdicas? (5a. Ed.). Valencia,España, España: Universidad Politécnica de Valencia. 
Novelo, F. (2000). La política exterior de México, en la era de la globalización (Disertación doctoral, Universidad Autónoma Metropolitana, 2000).

Organización para la Cooperación y Desarrollo Económico (2010). Pagina web. Recuperado el 10 de noviembre de 2010, de: http://www.oecd.org

Paredes, O. y Loyola, R. (2006). El conocimiento y la innovación, los grandes ausentes para el desarrollo y la competitividad en México. Revista Reencuentro, 5, 045, 9-11

Pedroza, A. y Sánchez J. (2005).Proceso de innovación tecnológica en la pequeña y mediana empresa. (1a. Ed.).Guadalajara, Jal, México:Universidad de Gadalajara.

Ramírez, P. (2009). Vinculación entre ciencia y tecnología, empresa y emprendimiento. Implicaciones de gran trascendencia para la capacidad creativa y emprendedora del mexicano. Ide@s CONCYTEG. 47(4), 671-684.

Rivera, D. (2009). Barreras y oportunidades para la innovación tecnológica en las empresas. Ide@s CONCYTEG. 50(4),685-691.

Secretaría de Hacienda y Crédito Público (2008). Avances y retos en materia de competitividad. Recuperado el 15 de noviembre de 2010, de: http://www.scribd.com/doc/7505506/Informe-Semanal-42-del-Vocero-de-la- SHCP 